Trapped atoms in one-dimensional photonic crystals

This article has been downloaded from IOPscience. Please scroll down to see the full text article.

2013 New J. Phys. 15083026

(http://iopscience.iop.org/1367-2630/15/8/083026)

View the table of contents for this issue, or go to the journal homepage for more

Download details:

IP Address: 131.215.71.79

The article was downloaded on 05/09/2013 at 16:03

Please note that terms and conditions apply. 


\title{
Trapped atoms in one-dimensional photonic crystals
}

\author{
C-L Hung ${ }^{1,2,5}$, S M Meenehan ${ }^{2,3,5}$, D E Chang ${ }^{4}$, O Painter ${ }^{2,3}$ \\ and $\mathbf{H} \mathbf{J}$ Kimble ${ }^{1,2,6}$ \\ ${ }^{1}$ Norman Bridge Laboratory of Physics 12-33, California Institute of \\ Technology, Pasadena, CA 91125, USA \\ ${ }^{2}$ Institute for Quantum Information and Matter, California Institute of \\ Technology, Pasadena, CA 91125, USA \\ ${ }^{3}$ Thomas J Watson, Sr, Laboratory of Applied Physics, California Institute of \\ Technology, Pasadena, CA 91125, USA \\ ${ }^{4}$ ICFO_Institut de Ciencies Fotoniques, Mediterranean Technology Park, \\ E-08860 Castelldefels, Barcelona, Spain \\ E-mail: hjkimble@caltech.edu
}

New Journal of Physics 15 (2013) 083026 (15pp)

Received 26 April 2013

Published 9 August 2013

Online at http://www.njp.org/

doi:10.1088/1367-2630/15/8/083026

\begin{abstract}
We describe one-dimensional (1D) photonic crystals that support a guided mode suitable for atom trapping within a unit cell, as well as a second probe mode with strong atom-photon interactions. A new hybrid trap is analyzed that combines optical and Casimir-Polder forces to form stable traps for neutral atoms in dielectric nanostructures. By suitable design of the band structure, the atomic spontaneous emission rate into the probe mode can exceed the rate into all other modes by more than tenfold. The unprecedented single-atom reflectivity $r_{0} \gtrsim 0.9$ for the guided probe field should enable diverse investigations of photon-mediated interactions for 1D atom chains and cavity quantum electrodynamics.
\end{abstract}

\footnotetext{
${ }^{5}$ These authors contributed equally to this research.

${ }^{6}$ Author to whom any correspondence should be addressed.
}

Content from this work may be used under the terms of the Creative Commons Attribution 3.0 licence. Any further distribution of this work must maintain attribution to the author(s) and the title of the work, journal citation and DOI. 


\section{Contents}

\section{Introduction}

2. Design principles for the one-dimensional photonic crystal waveguides 4

3. Optical trapping and surface Casimir-Polder potential inside the 1D-waveguides 4

3.1. Stable optical trapping in a single nanobeam . . . . . . . . . . . . 6

3.2. A new hybrid trap in double nanobeams: utilizing vacuum Casimir-Polder force to realize a stable trap condition . . . . . . . . . . . . . 6

4. Achieving strong atom-photon interactions via guided modes near a photonic band edge

4.1. Green's tensor and atomic spontaneous emission inside the 1D-waveguide . . . 7

4.2. Enhanced probe-mode coupling rate $\gamma_{1 \mathrm{D}}$ and the resulting strong atom-photon interaction . . . . . . . . . . . . . . . . 8

5. Conclusion

Acknowledgment

Appendix A. Calculation of Casimir-Polder potentials 10

Appendix B. Calculation of $\gamma_{\text {tot }} \quad 12$

Appendix C. Validation $\quad 12$

Appendix D. Guided mode resonances $\quad 13$

Appendix E. Effective area and mode volume for probe $\quad 14$

$\begin{array}{ll}\text { References } & 14\end{array}$

\section{Introduction}

New opportunities in atomic, molecular and optical (AMO) physics and quantum information science emerge from the capability to achieve strong radiative interactions between single atoms and the fields of nanoscopic optical waveguides and resonators [1]. For example, strong atom-photon interactions in lithographic structures [2-6] could be used to create quantum optical circuits with long-range atom-atom interactions mediated by single photons [7, 8]. Moreover, linear arrays of atoms radiatively coupled to nanophotonic waveguides exhibit a wide range of remarkable phenomena, including coherent transport of atomic emission [9-11], guided superradiance and polaritons [12-14], as well as highly reflecting atomic mirrors $[15,16]$. The interplay of atomic emission into the waveguide and photonmediated forces can lead to self-organization of atoms into exotic spatial configurations along the waveguide $[17,18]$.

A long-standing obstacle to this scientific frontier is the challenge of trapping atoms in vacuum near dielectric surfaces $(\sim 100 \mathrm{~nm})$ while at the same time achieving strong interactions between one atom and photon. A far-off resonance dipole-force trap (FORT) [19] can provide atomic localization by using modes of the dielectric for optical trapping [20-22] and has been used to trap cold atoms within hollow-core optical fibers [23-26] and external to fiber-taper waveguides [27-29].

Motivated by these advances, in this paper we present principles for the design of optical traps and strong atom-photon interactions in one-dimensional (1D) photonic crystal 


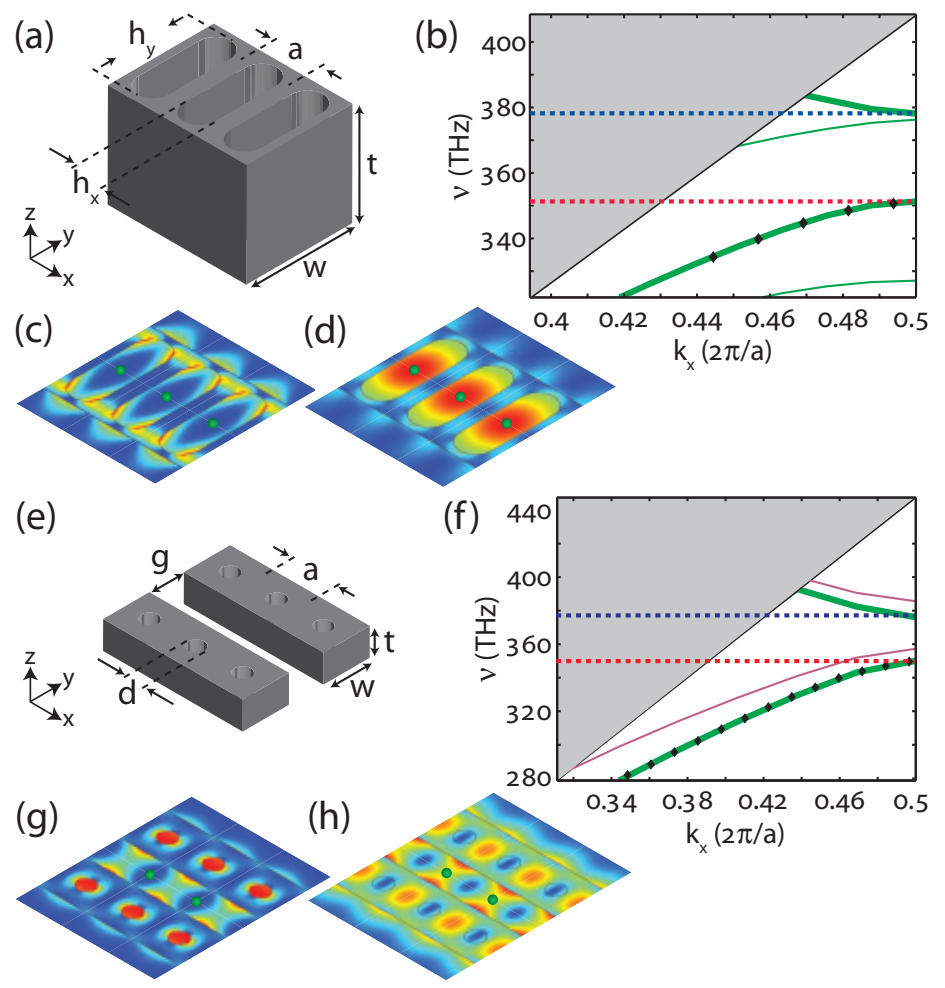

Figure 1. (a) Schematic for the single nanobeam structure with dimensions $\left(a, w, t, h_{x}, h_{y}\right)=(367,845,825,246,745) \mathrm{nm}$. (b) Band diagram for the single nanobeam in (a) showing only bands with even vector symmetry about the $y$ and $z$ symmetry planes. The trapping and probing bands are shown as thicker lines, with the trap $\omega_{\mathrm{T}} / 2 \pi$ (probe $\omega_{\mathrm{A}} / 2 \pi$ ) frequency as a blue (red) dashed line. (c) Field intensity of the blue trapping mode and (d) field amplitude of the probe mode in the center plane $z=0$ for the single nanobeam in (a). Green spheres mark the locations of minima of the trapping potential. (e) Schematic for the double nanobeam structure with $(a, w, t, d, g)=(335,335,200,116,250) \mathrm{nm}$. (f) Band diagram for the double nanobeams in (e) displaying only modes of even vector symmetry in $z$. The proximity of the two nanobeams results in a band structure composed of even (green) and odd (magenta) superpositions of single nanobeam modes. We focus on the even parity supermodes due to their large field amplitude in the gap. (g) Field intensity of the blue trapping mode and (h) field amplitude of the probe mode in the center plane $z=0$ of the double nanobeams in (e). The black diamonds in (b) (f) mark resonances for finite structures of 81 unit cells from figures 4 and 5).

waveguides. Our choice of 1D waveguides is based on their simple photonic band structures and their compatibility with current laser cooling and trapping technology [27-29].

We analyze the trap potential $U_{\text {tot }}(\mathbf{r})$ due to light-shifts from a FORT [30-32] together with Casimir-Polder (CP) interactions with the dielectric [33-36] (figures 1(a) and (e)). Despite the proximity of the surfaces, stable potentials $U_{\text {tot }}(\mathbf{r})$ are achieved for modest optical intensities $\left(\sim 5 \mathrm{~mW} \mu \mathrm{m}^{-2}\right)$ for blue-detuned FORTs operated at a 'magic' wavelength for 
the D2 line of atomic cesium [19]. A new possibility for trapping is also identified for which vacuum forces from $\mathrm{CP}$ interactions are exploited to close the trap perpendicular to the plane of structure, which would otherwise be unstable with either the FORT or the CP potential alone [37].

In addition to the waveguide trapping properties, strong near-resonance atom-photon interactions of trapped atoms are found to arise for waveguides with properly tailored band structure [38-42]. For practically realizable structures, we find $\gamma_{1 \mathrm{D}} / \gamma^{\prime} \gtrsim 10$, where $\gamma_{1 \mathrm{D}}$ is the atomic decay rate into the (guided) probe mode and $\gamma^{\prime}$ the rate into all other modes. One atom trapped within the structure could thereby attenuate a resonant probe with transmission $\left|1-r_{0}\right|^{2} \lesssim 10^{-2}[9,16]$.

\section{Design principles for the one-dimensional photonic crystal waveguides}

As illustrated in figure 1, we focus on two of the simplest quasi-1D photonic crystal geometries. The first waveguide consists of a single silicon-nitride nanobeam (refractive index $n=2$ ) with a 1D array of filleted rectangular holes along the propagation direction; atoms are trapped in the centers of the holes (figure 1(a)). The second waveguide consists of two parallel silicon nitride nanobeams, each with a periodic array of circular holes, with atoms trapped in the gap between the beams (figure 1(e)).

The design of a 1D-photonic crystal waveguide with distinct modes for optical trapping and strong atom-photon interactions is constrained by the region of the optical band structure containing a continuum of unguided optical modes (i.e. the light cone indicated in gray in figures 1(b) and (f)). Modes within the light cone can still have large amplitude in the structure but radiate energy into the surrounding vacuum leading to unacceptable loss. The top of the vacuum light line is at the Brillouin zone boundary $\left(X\right.$-point, where $\left.k_{x} a=\pi\right)$, so the lattice constant $a$ is constrained by $a<\lambda / 2$, where $\lambda$ is the smaller of the (vacuum) wavelengths for trapping and probe fields. Here, $k_{x}$ is the Bloch wavevector along the waveguide axis $x$.

Once $a$ is fixed, additional guided modes can be 'pulled' below the light line by increasing the width and thickness of the structure. With appropriate modes below the light line for probing and trapping, the spacing of these modes at the $X$-point can be tuned by altering the size of the holes, which enables the probe mode to be resonant with the frequency $\omega_{\mathrm{A}}$ of the atomic transition while simultaneously matching the optical frequency $\omega_{\mathrm{T}}$ of the trap mode to a 'magic' frequency for the atom [19].

\section{Optical trapping and surface Casimir-Polder potential inside the 1D-waveguides}

Within this general context, here we consider only blue-detuned FORTs for which the trapping mode has an intensity minimum at the trapping site ${ }^{7}$. Our analysis is for the $D 2$ line of atomic Cs with probe wavelength near the atomic resonance $\lambda_{\mathrm{A}}=852 \mathrm{~nm}$ and with a blue-detuned FORT at the magic wavelength $\lambda_{\mathrm{T}}=793 \mathrm{~nm}$ [19]. Note that our results are readily transcribed to other atomic transitions by way of the scale invariance of Maxwell's equations [43].

The photonic crystals are assumed to be suspended in vacuum and composed of SiN. Band structures are calculated using the MIT photonic-bands (MPB) software package [44].

7 We have also designed structures for trap and probe modes for red-detuned FORTs with an atom trapped at the intensity maximum in a unit cell. 


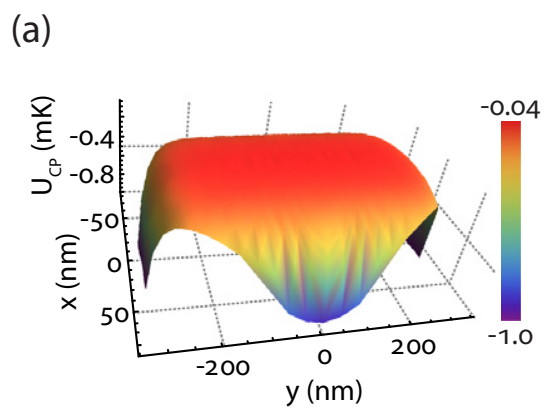

(b)

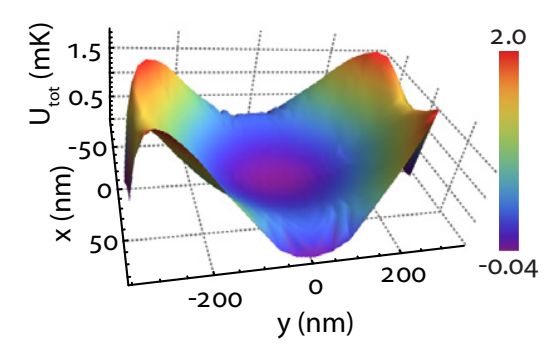

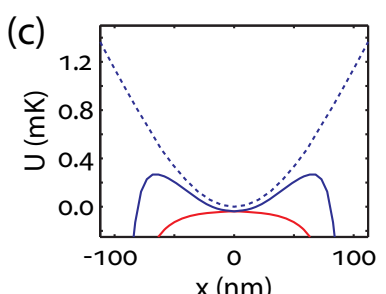
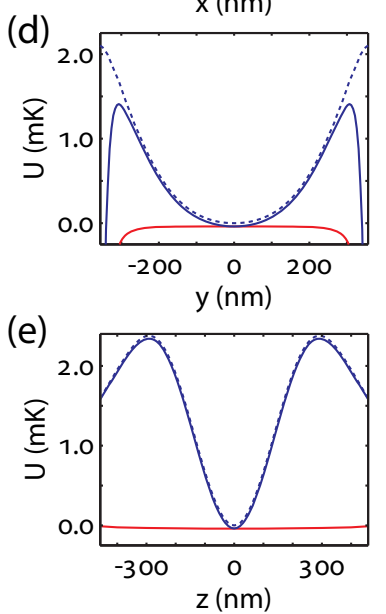

Figure 2. Trapping potentials for the single nanobeam structure in figure 1(a) for Cs $6 \mathrm{~S}_{1 / 2}, F=4$ level and $\lambda_{\mathrm{T}}=793 \mathrm{~nm}$. (a) CP potential $U_{\mathrm{CP}}(\mathbf{r})$ and (b) total potential $U_{\mathrm{tot}}(\mathbf{r})=U_{\mathrm{CP}}(\mathbf{r})+U_{\mathrm{FORT}}(\mathbf{r})$ in the central $z=0$ plane. Panels (c)-(e) show line cuts of $U_{\mathrm{CP}}$ (red solid), $U_{\mathrm{FORT}}$ (blue dashed) and $U_{\text {tot }}$ (blue solid) along the (c) $x$-, (d) $y$ - and (e) $z$-axis. Average trap intensity for a unit cell is $4.9 \mathrm{~mW} \mu \mathrm{m}^{-2}$.

Field profiles for guided modes are calculated using the finite-element-method simulations ${ }^{8}$. Results for the single and double nanobeam structures are presented in figure 1.

With suitable guided modes for trapping in hand, we have developed numerical tools for evaluating the FORT and CP potentials inside the waveguide, and hence the total potential $U_{\mathrm{tot}}=U_{\mathrm{FORT}}+U_{\mathrm{CP}}$. The adiabatic potential $U_{\mathrm{FORT}}(\mathbf{r})$ is readily calculated using the electric field distribution of the trap mode, $\mathbf{E}(\mathbf{r})=\mathbf{u}_{k_{x}}(\mathbf{r}) \mathrm{e}^{\mathrm{i} k_{x} x}$ [30-32]; $\mathbf{u}_{k_{x}}(\mathbf{r})$ is the periodic Bloch wave function at $k_{x}$.

The surface potential $U_{\mathrm{CP}}\left(\mathbf{r}_{a}\right)$ is determined from the formalism in [35] for the imaginary component of the scattering Green's tensor $\mathbf{G}_{\mathrm{sc}}\left(\mathbf{r}_{\mathrm{a}}, \mathbf{r}_{\mathrm{a}}, \omega\right)$ from Maxwell's equations for a point dipole at the atomic location $\mathbf{r}_{\mathrm{a}}$ with the vacuum contribution (i.e. no dielectric structure) subtracted. We evaluate $\mathbf{G}_{\mathrm{sc}}\left(\mathbf{r}_{\mathrm{a}}, \mathbf{r}_{\mathrm{a}}, \omega\right)$ numerically by adapting the procedures from [36] (see appendix A).

Figures 2 and 3 display numerical results for $U_{\mathrm{CP}}(\mathbf{r}), U_{\mathrm{FORT}}(\mathbf{r})$ and $U_{\text {tot }}(\mathbf{r})$ for the single and double nanobeams for $k_{x}$ below the $X$-point. The calculations are for the $6 \mathrm{~S}_{1 / 2}, F=4$ hyperfine ground state of $\mathrm{Cs}$ for the FORT modes indicated in figures $1(\mathrm{c})$ and $(\mathrm{g})^{9}$. For these

8 COMSOL Multiphysics (http://comsol.com/).

$9 U_{\text {tot }}(\mathbf{r})$ is largely insensitive to the particular atomic Zeeman sublevel owing to negligible vector shifts due to the high degree of linear polarization of the trapping modes and to the absence of ground state tensor polarizability. 
(a)

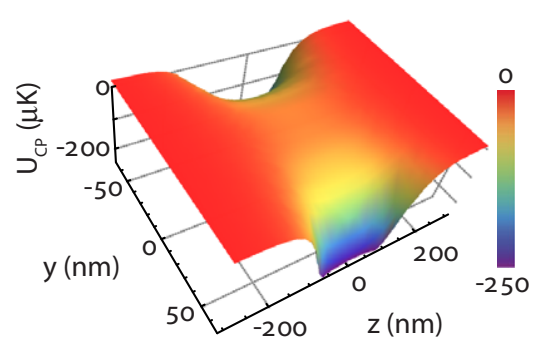

(b)

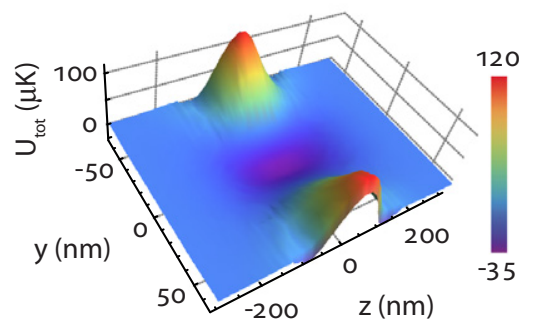

(c)

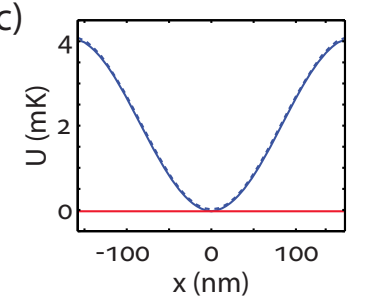

(d)

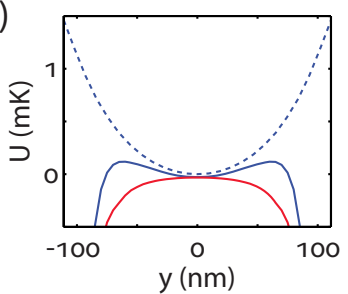

(e)

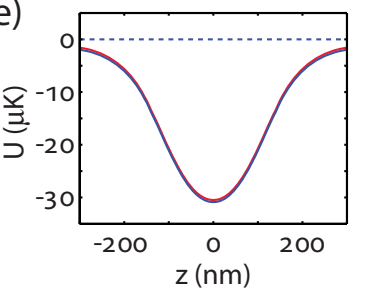

Figure 3. Trapping potentials for the double nanobeam structure in figure 1(e) for Cs $6 \mathrm{~S}_{1 / 2}, F=4$ level and $\lambda_{\mathrm{T}}=793 \mathrm{~nm}$. (a) CP potential $U_{\mathrm{CP}}(\mathbf{r})$ and (b) total potential $U_{\text {tot }}(\mathbf{r})=U_{\mathrm{CP}}(\mathbf{r})+U_{\mathrm{FORT}}(\mathbf{r})$ in the transverse $x=0$ plane. Panels (c)-(e) show line cuts of $U_{\mathrm{CP}}$ (red solid), $U_{\mathrm{FORT}}$ (blue dashed) and $U_{\text {tot }}$ (blue solid) along the (c) $x$-, (d) $y$ - and (e) $z$-axis. Average trap intensity for a unit cell is $3.5 \mathrm{~mW} \mu \mathrm{m}^{-2}$.

initial calculations, we make the reasonable assumption for $\operatorname{SiN}$ that the dielectric constant $\epsilon$ is frequency independent, $\epsilon(\mathbf{r}, \omega) \rightarrow \epsilon(\mathbf{r})$; see appendix A.1 for the justification by numerical evaluation of $U_{\mathrm{CP}}(\mathbf{r})$ using the full frequency dependence of $\epsilon(\omega)$ for SiN at several locations.

\subsection{Stable optical trapping in a single nanobeam}

$U_{\text {tot }}(\mathbf{r})$ for the single nanobeam in figure 2 reveals that modest optical intensity is sufficient to overcome the attractive CP interactions and create a stable potential in the center of the vacuum space at $\mathbf{r}_{\min }=0$ within a unit cell. An atom would be localized at distances $\left(d_{x}, d_{y}\right)=$ $(123,373) \mathrm{nm}$ from the walls of the dielectric. The trap oscillation frequencies for a Cs atom would be $\left(f_{x}, f_{y}, f_{z}\right) \simeq(612,180,484) \mathrm{kHz}$.

\subsection{A new hybrid trap in double nanobeams: utilizing vacuum Casimir-Polder force to realize} a stable trap condition

For the double nanobeams [45], the FORT alone is insufficient to trap the atom, as the mode has a (weak) local intensity maximum along the $z$ direction that repels an atom. However, the CP potential $U_{\mathrm{CP}}(\mathbf{r})$ along $z$ provides the force necessary to overcome the repulsive optical force 
and to form a stable trap. The result is a hybrid optical-vacuum trap that circumvents the 'no-go' theorem for vacuum trapping alone [37].

Potentials for our hybrid trap are illustrated in figure 3. At the trap minimum $\mathbf{r}_{\min }=0$, an atom would be localized at distance $d_{y}=125 \mathrm{~nm}$ from adjacent surfaces of the dielectric beams. Oscillation frequencies for a Cs atom would be $\left(f_{x}, f_{y}, f_{z}\right) \simeq(1013,390,57) \mathrm{kHz}$.

\section{Achieving strong atom-photon interactions via guided modes near a photonic band edge}

As concerns strong radiative interactions, our structures trap an atom in a region of large amplitude for the probe field, leading to small mode volume per unit cell (appendix E). Atom-photon interactions can be further enhanced near a band edge [38-42], where the density of states diverges due to a van Hove singularity. To quantify the radiative coupling, we determine the decay rate $\gamma_{\text {tot }}$ for a point dipole located at $\mathbf{r}_{\mathrm{a}}=0$ for a structure with $N$ unit cells ${ }^{10}$. Finite-difference-time-domain (FDTD) calculations are performed to evaluate the classical Green's tensor $\mathbf{G}\left(\mathbf{r}_{\mathrm{a}}, \mathbf{r}_{\mathrm{a}}, \omega\right)$ and thence $\gamma_{\text {tot }}$ following [34, 35, 46] (appendix B).

\subsection{Green's tensor and atomic spontaneous emission inside the 1D-waveguide}

Figures 4(a) and 5(a) display the diagonal components $\operatorname{Im}\left[G_{i i}\left(v_{\mathrm{d}}\right)\right]$ of the Green's tensor as functions of dipole frequency $v_{\mathrm{d}}=\omega_{\mathrm{d}} / 2 \pi$ and relate to the emission rate of resonant point dipoles polarized along the $i=x-, y$ - or $z$-axis for the single and double nanobeams. Firstly, in figure 4(a), $\operatorname{Im}\left[G_{i i}\right]$ is enhanced along the $x$ (periodic) direction across a broad frequency range, and is suppressed in the $y$ and $z$ directions, as can be explained by the orientation of the induced array of image dipoles along the single nanobeam.

Secondly, figures 4(a) and 5(a) display a series of resonant peaks due to strong emission into various guided modes. In the region near the Cs $D 2$ line (i.e. $v_{\mathrm{d}} \simeq v_{\mathrm{A}}=\omega_{\mathrm{A}} / 2 \pi=352 \mathrm{THz}$ ), we find peaks in $\operatorname{Im}\left[G_{x x}\right]$ for the single nanobeam and in $\operatorname{Im}\left[G_{y y}\right]$ for the double nanobeams. These peaks are due to emission into our designated probe modes for the respective structures, where for the single (double) nanobeam(s), the probe mode is principally polarized along the $x$ - $(y-)$ axis. Each peak is from a discrete set of propagation constants $k_{x}^{(n)} \simeq \pi n / a N$ imposed by the boundary conditions for the finite structures. Here, $N$ is the total number of cells in the single (double) beam, and $n \leqslant N$ is an even (odd) integer. We find excellent agreement between the frequencies of these resonances and the band diagram of the probe mode ('diamonds' in figures 1(b) and (f)) for various $(n, N)$.

The peaks become larger and narrower as $k_{x}^{(n)}$ approaches the $X$-point, owing to the diminishing group velocity $[40,42]$. Beyond the $X$-point, the probe resonances disappear, leaving a broad background corresponding to coupling into lossy (radiation) modes.

On an expanded frequency scale around $v_{\mathrm{d}} \simeq v_{\mathrm{A}}$, figures 4(b) and 5(b) show calculated atomic decay rates $\gamma_{\text {tot }}$ for the $6 \mathrm{P}_{3 / 2}, \mathrm{~F}^{\prime}=5 \rightarrow 6 \mathrm{~S}_{1 / 2}, \mathrm{~F}=4$ transition in atomic Cs (appendix B). When the atomic dipole is aligned along the principal polarization of the designated probe mode ( $\hat{x}$ - single beam; $\hat{y}$ - double beam), the emission rate $\gamma_{1 \mathrm{D}}$ into the probe mode is strongly

\footnotetext{
${ }^{10}$ A finite number of unit cells is chosen to better represent an actual structure. Since our focus is not on specific boundaries, the structure is terminated by vacuum.
} 

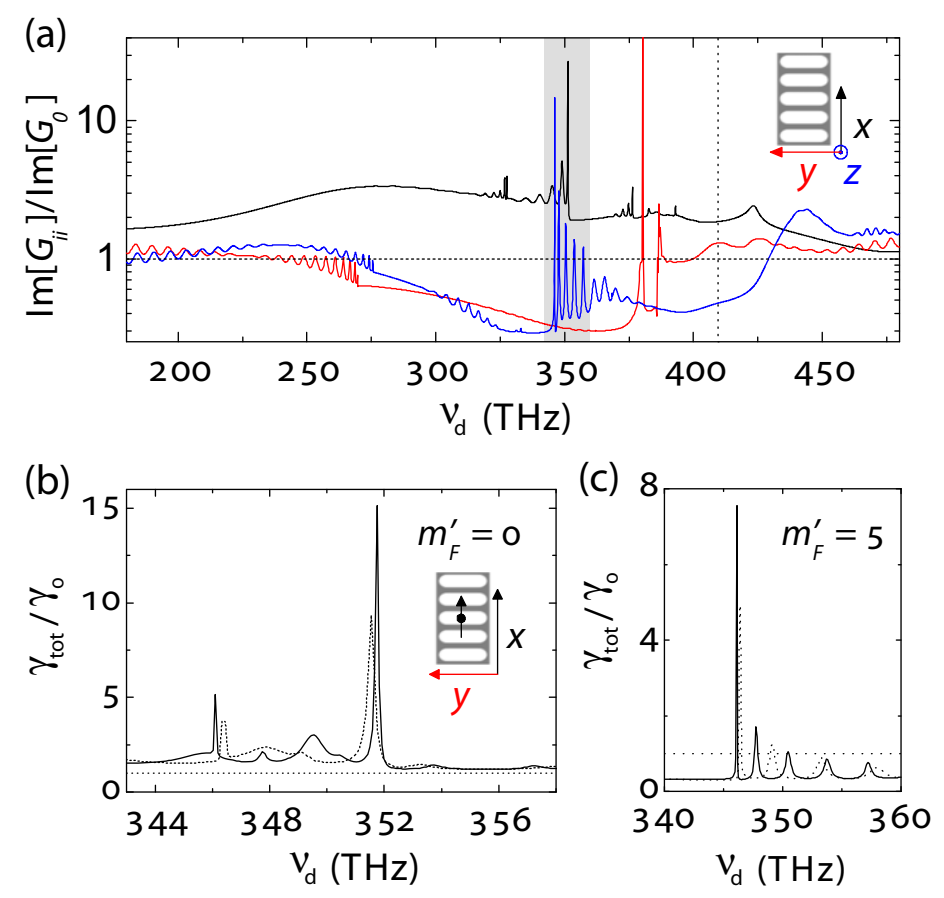

Figure 4. Green's tensor and total atomic decay rate $\gamma_{\text {tot }}$ versus source dipole frequency $v_{d}$ for the single nanobeam at $\mathbf{r}=0$. Panel (a) shows the diagonal components of the Green's tensor, $\operatorname{Im}\left[G_{x x}\right]$ (solid black), $\operatorname{Im}\left[G_{y y}\right]$ (red) and $\operatorname{Im}\left[G_{z z}\right]$ (blue), normalized to the free space value $\operatorname{Im}\left[G_{0}\right]$ (dashed line). The number of unit cells is $N=81$. Cesium $D 2$-line frequency $v_{\mathrm{A}}=352 \mathrm{THz}$ is centered in the shaded area. The vertical dotted line marks the light line, beyond which all decay channels are lossy. Panels (b) and (c) show $\gamma_{\text {tot }}$ (black curves), normalized to the free space value $\gamma_{0}$ (dotted line), in the frequency range marked by the shaded area in (a). The solid (dashed) curve is evaluated using 81(61) unit cells. The atomic spin is aligned to the $x$-axis, with the spin projection quantum number (b) $m_{\mathrm{F}}^{\prime}=0$ and (c) $m_{\mathrm{F}}^{\prime}=5$.

enhanced at frequencies corresponding to $k_{x}^{(n)}$ near the $X$-point. Specifically, for $v_{\mathrm{d}}=v_{\mathrm{A}}$ large enhancements in $\gamma_{1 \mathrm{D}}$ occur for the initial excited state $6 \mathrm{P}_{3 / 2}, \mathrm{~F}^{\prime}=5, m_{\mathrm{F}}^{\prime}=0$, while $\gamma_{1 \mathrm{D}}$ is suppressed for the initial state $m_{\mathrm{F}}^{\prime}=5$. This is because the probe mode predominantly supports $\pi$-polarization and hence $\Delta m_{\mathrm{F}}=0$. Coupling between states with $\Delta m_{\mathrm{F}} \neq 0$ is small. Of course, additional guided modes can contribute to $\gamma_{\text {tot }}$, as is evidenced for the $m_{\mathrm{F}}^{\prime}=5$ state due to field polarizations perpendicular to the atomic spin, such as $\hat{z}(\hat{x})$ for the single (double) beam(s) in figure 4(a) (figure 5(a)).

\subsection{Enhanced probe-mode coupling rate $\gamma_{1 \mathrm{D}}$ and the resulting strong atom-photon interaction}

From $\gamma_{\text {tot }}\left(\nu_{\mathrm{d}}\right)$ and an analytic model of coupling to the guided-mode near the $X$-point, we estimate the contributions of $\gamma_{1 \mathrm{D}}$ and $\gamma^{\prime}$ to $\gamma_{\text {tot }}=\gamma_{1 \mathrm{D}}+\gamma^{\prime}$ near the largest resonances in figures 4(b) and 5(b) (appendix D.1). For $m_{\mathrm{F}}^{\prime}=0$ and $N=81$, we find that $\gamma_{1 \mathrm{D}} / \gamma_{0} \simeq 15$ 

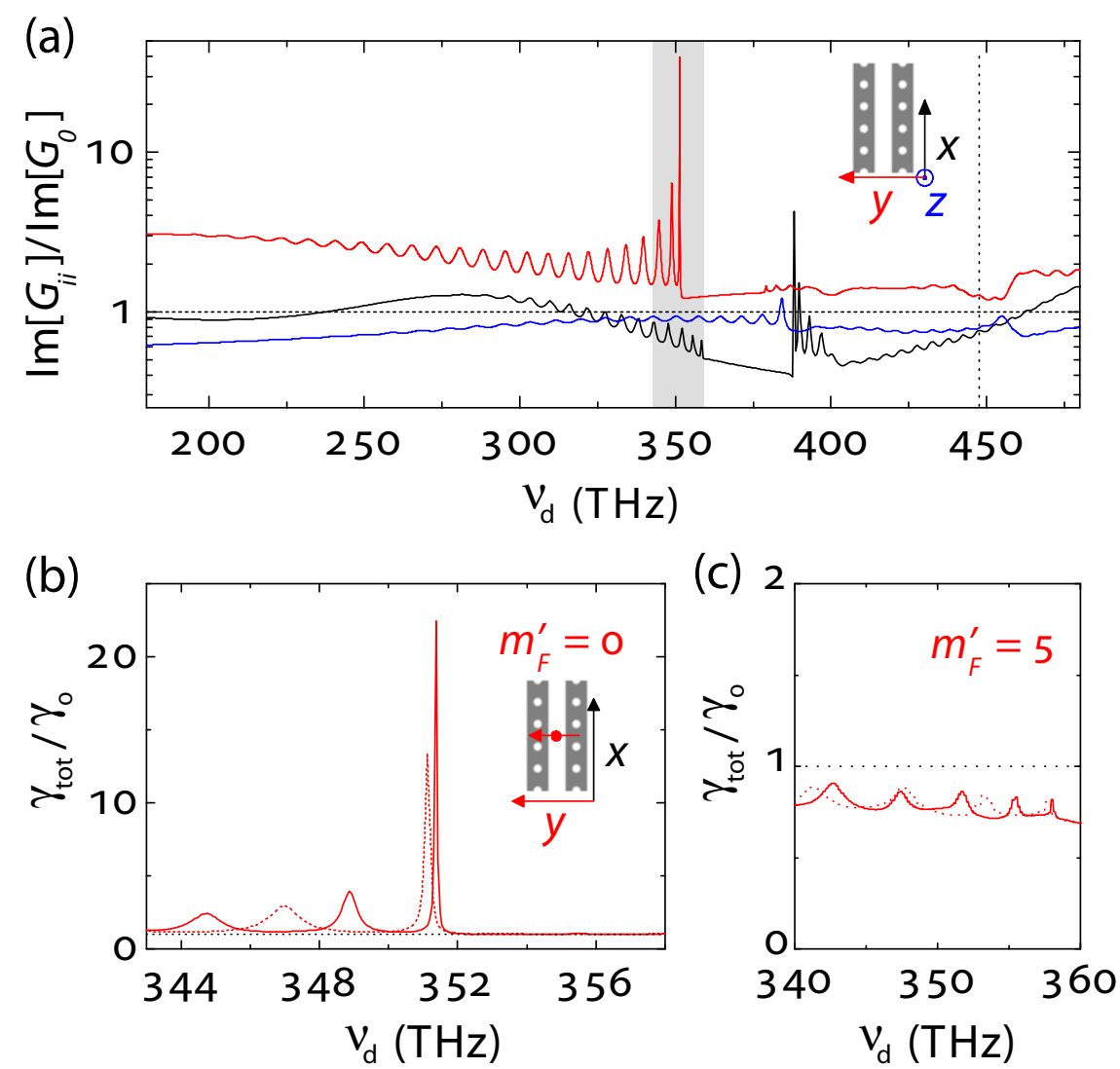

Figure 5. As in figure 4 now for the double nanobeams at $\mathbf{r}=0$. Panel (a) shows the diagonal components of the Green's tensor, $\operatorname{Im}\left[G_{x x}\right]$ (solid black), $\operatorname{Im}\left[G_{y y}\right]$ (red), and $\operatorname{Im}\left[G_{z z}\right]$ (blue), normalized to $\operatorname{Im}\left[G_{0}\right]$ for free space (dashed line). $N=81$ unit cells. Cesium D2-line frequency $v_{\mathrm{A}}=352 \mathrm{THz}$ is centered in the shaded area. The vertical dotted line marks the light line, beyond which all decay channels are lossy. Panels (b) and (c) show $\gamma_{\text {tot }}$ (red curves), normalized to the free space value $\gamma_{0}$ (dotted line), in the frequency range marked by the shaded area in (a). The solid (dashed) curve is evaluated using 81(61) unit cells. The atomic spin is aligned to the $y$-axis, with the spin projection quantum number (b) and (c) $m_{F}^{\prime}=5$.

and $\gamma^{\prime} / \gamma_{0} \simeq 1.2$ for the single nanobeam, while $\gamma_{1 \mathrm{D}} / \gamma_{0} \simeq 21$ and $\gamma^{\prime} / \gamma_{0} \simeq 1.0$ for the double nanobeams ${ }^{11}$. Here, $\gamma_{0} / 2 \pi=5.2 \mathrm{MHz}$, the free-space Cs decay rate.

The ratios $\gamma_{1 \mathrm{D}} / \gamma_{\mathrm{tot}}$ and $\gamma_{1 \mathrm{D}} / \gamma^{\prime}$ serve as metrics for the strength of atom-photon interactions for our 1D photonic crystals. For example, the resonant reflectivity $r_{0}$ of a trapped atom for the probe field should scale as $r_{0}=\gamma_{1 \mathrm{D}} / \gamma_{\text {tot }}[9,16]$, which for the double nanobeams leads to $r_{0} \simeq 0.95$. For a cavity quantum electrodynamics (QED) system with one 'impurity' atom surrounded by $N_{\mathrm{A}}$ 'mirror' atoms along a 1D-lattice [16], the ratio of the coherent coupling rate $g_{1}=\sqrt{N_{\mathrm{A}}} \gamma_{1 \mathrm{D}} / 2$ to the effective dissipative rate $\gamma^{\prime}$ would exceed unity even for $N_{\mathrm{A}}=1$ atom. For conventional cavity QED, we estimate a 1-photon Rabi frequency $\sim 2 \pi \times 2 \mathrm{GHz}$ for figure 5(b) with $N=81$.

${ }^{11}$ For comparison, $\gamma_{1 \mathrm{D}} / \gamma_{0} \simeq 0.06$ is the state of the art for a Cs atom trapped near an $\mathrm{SiO}_{2}$ nanofiber [29]. 


\section{Conclusion}

Certainly there are challenges in the implementation of our designs, including atom loading into the small trap volumes and light scattering from device imperfections. The latter can reduce the expected coupling rate $\gamma_{1 \mathrm{D}}$ and introduce trap imperfections. The former challenge may be resolved either by loading atoms continuously from an atomic reservoir surrounding the nanostructure [27-29] to improve the loading efficiency, or by using a moving optical lattice to transfer atoms precisely into the trapping sites. We are working to address these issues by numerical simulation, device fabrication and cold-atom experiments with nanoscopic structures. Our efforts are motivated by the prediction $\gamma_{1 \mathrm{D}} / \gamma_{\text {tot }} \gtrsim 0.9$ in figures 4 and 5 , which is unprecedented in AMO physics and which could create new scientific opportunities (e.g. quantum many-body physics for 1D atom chains with photon-mediated interactions, and highprecision studies of vacuum forces). Moreover, our double nanobeam structure provides proofof-principle for a promising new concept that combines optical and vacuum forces to form stable traps for neutral atoms in dielectric nanostructures.

Our designs of $1 \mathrm{D}$ periodic structures represent a basis for extensions to more complicated geometries, including two-dimensional (2D) photonic crystals with trapped atoms. A step in this direction is with structures that are still 1D in nature (e.g. a line of defect patterns embedded in a $2 \mathrm{D}$ photonic band gap crystal). Such structures should offer larger values for $\gamma_{1 \mathrm{D}}$ and further suppressions of emission rate $\gamma^{\prime}$ to other modes (including the vacuum).

\section{Acknowledgment}

We gratefully acknowledge D J Alton, K S Choi, D Ding and A Goban. Funding is provided by the IQIM, an NSF Physics Frontier Center with support of the Moore Foundation, by the AFOSR QuMPASS MURI, by the DoD NSSEFF program (HJK) and by NSF grant no. PHY0652914 (HJK). DEC acknowledges funding from Fundacio Privada Cellex Barcelona.

Note added in proof. After submission of our paper, related work in [47] was published.

\section{Appendix A. Calculation of Casimir-Polder potentials}

The CP potential $U_{\mathrm{CP}}\left(\mathbf{r}_{\mathrm{a}}\right)$ is calculated from the following integral [35]:

$$
U_{\mathrm{CP}}\left(\mathbf{r}_{\mathrm{a}}\right)=-\frac{\hbar \mu_{0}}{2 \pi} \operatorname{Im}\left\{\int_{0}^{\infty} \mathrm{d} \omega \omega^{2} \operatorname{Tr}\left[\boldsymbol{\alpha}^{0}(\omega) \cdot \mathbf{G}_{\mathrm{sc}}\left(\mathbf{r}_{\mathrm{a}}, \mathbf{r}_{\mathrm{a}}, \omega\right)\right]\right\},
$$

where $\operatorname{Tr}$ [.] denotes the trace, $\boldsymbol{\alpha}^{0}$ is the dynamic polarizability tensor of ground-state cesium atom, and $\mathbf{G}_{\mathrm{sc}}\left(\mathbf{r}_{\mathrm{a}}, \mathbf{r}_{\mathrm{a}}, \omega\right)=\mathbf{G}\left(\mathbf{r}_{\mathrm{a}}, \mathbf{r}_{\mathrm{a}}, \omega\right)-\mathbf{G}_{0}\left(\mathbf{r}_{\mathrm{a}}, \mathbf{r}_{\mathrm{a}}, \omega\right)$ is the scattering Green's tensor, that is, the Green's tensor $\mathbf{G}$ subtracted by the vacuum contribution $\mathbf{G}_{0}$ evaluated at atomic location $\mathbf{r}_{\mathrm{a}}$; $2 \pi \hbar$ is Planck's constant, and $\mu_{0}$ is the vacuum permeability. The Green's tensor is the solution to the Maxwell equation $\left[\nabla \times \nabla \times-\frac{\omega^{2}}{c^{2}} \epsilon(\mathbf{r}, \omega)\right] \mathbf{G}\left(\mathbf{r}, \mathbf{r}^{\prime}, \omega\right)=\mathbf{I} \delta^{(3)}\left(\mathbf{r}-\mathbf{r}^{\prime}\right)$, corresponding to the electric field response to a point dipole current source. $\epsilon(\mathbf{r}, \omega)$ is the dielectric function and $\mathbf{I}$ is the unity tensor.

We employ FDTD calculations [48] to solve numerically for the Green's tensors of our structures. The integral of equation (A.1) is evaluated by adapting a procedure established in [36] and by using a deformed contour $\omega(\xi)=\xi \sqrt{1+\mathrm{i} \sigma / \xi}$ in the upper half of the complex frequency plane, parameterized by a real number $\xi \geqslant 0$ and a constant $\sigma>0$. As 
explained in [36], this is equivalent to solving the Green's tensor at real frequencies $\xi$ with a fictitious global conductivity applied to the dielectric function $\epsilon^{\prime}(\mathbf{r}, \xi)=(1+\mathrm{i} \sigma / \xi) \epsilon(\mathbf{r}, \xi)$. The integration can then be performed in the time domain (via the convolution theorem) and converges quickly due to fast decay from $\sigma$.

Specifically, equation (A.1) is numerically evaluated using

$$
U_{\mathrm{CP}}\left(\mathbf{r}_{\mathrm{a}}\right)=\frac{\hbar}{2 \pi} \int_{0}^{\infty} \mathrm{d} t \operatorname{Im}\left[g_{\mu \nu}(-t)\right] \hat{x}_{\mu} \cdot \mathbf{E}_{\mathrm{sc}, v}\left(\mathbf{r}_{\mathrm{a}}, t\right),
$$

where $\mathbf{E}_{\mathrm{sc}, v}\left(\mathbf{r}_{\mathrm{a}}, t\right)$ is the (real) electric field generated by a point dipole current source $\mathcal{J}=\delta(t) \hat{x}_{v}$ $\left(\hat{x}_{v}=\hat{x}, \hat{y}, \hat{z}\right)$ located at the position $\mathbf{r}_{\mathrm{a}}$ and scattered by a structure with a dielectric function $\epsilon^{\prime}(\mathbf{r}, \xi) .{ }^{12}$ Here, the indices $\mu$ and $\nu$ are repeated for summation convention, $g_{\mu \nu}(t)$ is the Fourier transform of $g_{\mu \nu}(\xi)=-\mathrm{i} \xi \sqrt{1+\frac{\mathrm{i} \sigma}{\xi}}\left(1+\frac{\mathrm{i} \sigma}{2 \xi}\right) \Theta(\xi) \boldsymbol{\alpha}_{\mu \nu}^{0}(\omega(\xi))$ and $\Theta(\xi)$ is the Heaviside step function.

\section{A.1. Material dispersion}

For the initial calculations, we take the dielectric constant $\epsilon$ to be frequency independent, $\epsilon(\mathbf{r}, \omega) \rightarrow \epsilon(\mathbf{r})$. In principle it is important to take into account the frequency dependence of the refractive index when calculating the $\mathrm{CP}$ potential. However, for the trapping of atoms at distances of $100 \mathrm{~nm}$ from dielectric structures, the dominant contribution to the force comes from the material response near the atomic resonance frequencies. This is dictated by the smooth frequency cutoff coming from the atomic polarizability; see equation (A.1), as well as [49] for more detailed discussions. At distances on the order of the resonant wavelengths or more (not relevant to our calculations), the low-frequency material response would become increasingly important [49]. Since the refractive index of silicon nitride is approximately constant across the optical domain, we adopt the approximation based on a frequency independent dielectric constant $\epsilon(\mathbf{r})$.

In order to fully justify our assumption, we have performed calculations using full material dispersion, and have found the results well approximated by calculations using constant $n=2$. For the simulated material in FDTD calculations, we have taken the measured dispersion in dc, THz [50] and optical [51] regimes and use a Lorentz oscillator model to fit the complex refractive index $\tilde{n}=n+\mathrm{i} k$; see figures (A.1a)-(b). The resulting CP potentials are shown in figure A.1(c) for the single nanobeam structure and in figure A.1(d) for the double nanobeam structure. Each of them differs from the values calculated with $n=2$ by no more than $8 \%$ both at the trap center and closer to the walls.

The time required for computation increases linearly with the number of Lorentz oscillators used to model the material, and, for the nine oscillator model used here, the computation time increases by an order of magnitude. Due to limited computational resources, we have used the frequency independent results as a reasonable approximation when calculating the $\mathrm{CP}$ potential throughout both structures.

12 To obtain the CP potential for a single atom inside a periodic structure, we impose Bloch-periodic boundary conditions on a single unit-cell in the FDTD calculations. We then sum over all fields $\mathbf{E}_{\mathrm{sc}, v}\left(\mathbf{r}_{\mathrm{a}}, t ; k_{x}\right)$ with the Bloch wavevector $k_{x}$ across the first Brillouin zone to obtain $\mathbf{E}_{\mathrm{sc}, v}\left(\mathbf{r}_{\mathrm{a}}, t\right)=\frac{\mathrm{a}}{2 \pi} \int_{-\pi / \mathrm{a}}^{\pi / \mathrm{a}} \mathrm{d} k_{x} \mathbf{E}_{\mathrm{sc}, v}\left(\mathbf{r}_{\mathrm{a}}, t ; k_{x}\right)$, which is mathematically equivalent to solving for the fields of a single point dipole in an infinite periodic structure. The integral is approximated using a 10 point Gaussian quadrature. 


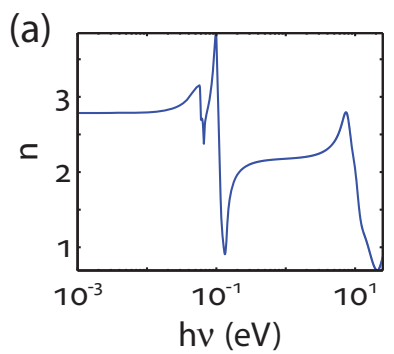

(c)

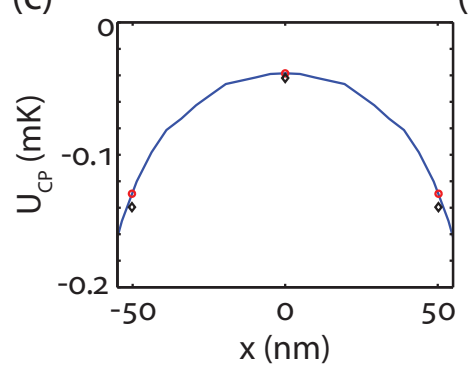

(b)

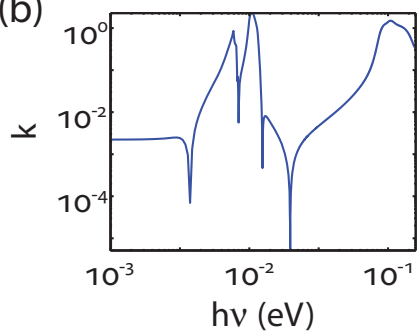

(d)

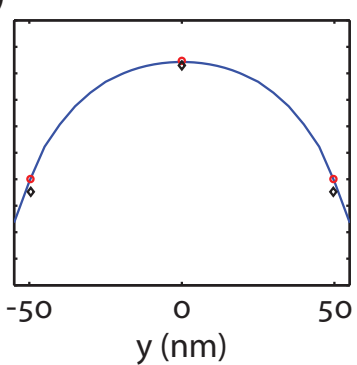

Figure A.1. Panels (a) and (b) show the real $(n)$ and imaginary $(k)$ refractive indices, respectively, of silicon nitride obtained from a model of 9 Lorentz oscillators. Panel (c) shows the CP potential calculated for the single nanobeam structure along the $x$-axis. The blue line shows the calculation using a frequency independent $n=2$. At the discrete points $x=-50,0$ and $50 \mathrm{~nm}$, the frequency independent results (red circles) were compared with results using the full frequency dependent material response (black diamonds). Panel (d) shows a similar plot along the $y$-axis of the double nanobeam structure.

\section{Appendix B. Calculation of $\gamma_{\text {tot }}$}

To determine the total spontaneous decay rate $\gamma_{\text {tot }}$ for an atom in our structures, we also solve for the classical Green's tensors and evaluate $\gamma_{\text {tot }}$ via $[34,35,46]$

$$
\gamma_{\text {tot }}=\frac{2 \mu_{0} \omega_{j}^{2}}{\hbar} \operatorname{Im}\left\{\sum_{\{0\}} \operatorname{Tr}\left[\mathbf{D}_{j} \cdot \mathbf{G}\left(\mathbf{r}_{\mathrm{a}}, \mathbf{r}_{\mathrm{a}}, \omega_{j}\right)\right]\right\},
$$

where $\mathbf{D}_{j}=\left\langle\{0\}\left|\mathbf{d}^{\dagger}\right| j\right\rangle\langle j|\mathbf{d}|\{0\}\rangle$ is the dipole matrix element between the ground state manifold and the excited state $j$, and $\omega_{j}$ is the transition frequency. The total decay rate $\gamma_{\text {tot }}=\gamma_{1 \mathrm{D}}+\gamma^{\prime}$ includes the decay rate $\gamma_{1 \mathrm{D}}$ to a guided mode of interest as well as the rate $\gamma^{\prime}$ to all other modes of the structure, including lossy modes. As discussed below, the contributions of $\gamma_{1 \mathrm{D}}, \gamma^{\prime}$ to $\gamma_{\text {tot }}$ can be estimated from the global frequency dependence $\gamma_{\text {tot }}(\omega)$.

To obtain $\gamma_{\text {tot }}(\omega)$, we evaluate the Green's tensor for the real dielectric function $\epsilon(\mathbf{r})$ using the FDTD method, followed by a discrete Fourier analysis.

\section{Appendix C. Validation}

To validate our numerical procedures, we have performed calculations of $U_{\mathrm{CP}}$ for several geometries where analytical solutions are available, including an atom near an infinite dielectric 
or metal half-space [52] and an atom located above an infinite dielectric grating [53], and found excellent agreement between our simulations and the exact results.

We have validated our calculations of $\gamma_{\text {tot }}$ for the cases of an (atomic) dipole near an infinite dielectric, metallic parallel plates, a nanofiber [54] and 2D-photonic band-gap microcavities [55].

\section{Appendix D. Guided mode resonances}

For our structure with an infinite number of unit cells, a guided mode (denoted by $\lambda$ ) contribution to the imaginary part of the Green's tensor can be calculated as $[46,56], \operatorname{Im}\left[\mathbf{G}_{1 \mathrm{D}}^{\lambda}\left(\mathbf{r}_{\mathrm{a}}, \mathbf{r}_{\mathrm{a}}, \omega\right)\right]=$ $a c^{2} \mathbf{u}_{\lambda}\left(\mathbf{r}_{\mathrm{a}} ; k_{x}\right) \otimes \mathbf{u}_{\lambda}^{*}\left(\mathbf{r}_{\mathrm{a}} ; k_{x}\right) / 2 \omega v_{\lambda}$, when the frequency $\omega$ intersects the frequency band $\omega_{\lambda}$ at a propagation constant $k_{x}$ below the light line. Here, $\mathbf{u}_{\lambda}\left(\mathbf{r} ; k_{x}\right)$ is the orthonormal mode function, and $v_{\lambda}$ is the group velocity, both available via numerical calculations [44] (see also footnote 8). As we scan the frequency $\omega, \operatorname{Im}\left[\mathbf{G}_{1 \mathrm{D}}^{\lambda}\right]$ increases monotonically and diverges as $k_{x}$ approaches the $X$-point, where $v_{\lambda} \rightarrow 0$. The guided mode Green's tensor vanishes when $\omega$ lies beyond the frequency of the band edge.

Based on this analysis, we can evaluate the decay rate $\gamma_{1 \mathrm{D}}^{(\infty)}(\omega)$ into the designated probe mode for an infinite structure, and compare it with the heights of resonant features in $\gamma_{\text {tot }}(\omega)$ for finite structures with different numbers of unit cells, as shown in figures 4(b) and 5(b). Indeed, the actual $\gamma_{1 \mathrm{D}}$ of a finite-size structure must deviate from $\gamma_{1 \mathrm{D}}^{(\infty)}$ due to boundary conditions that transform a continuous spectrum into a discrete set of resonant peaks [40], as shown in figures 4(b) and 5(b). When the number of unit cells is increased in our calculation of $\gamma_{1 \mathrm{D}}$ over the range $N=11$ to 81 , we find that the frequencies $\omega^{(n)}$ of the resonant peaks shift in position and the peaks change height. As documented by the black diamonds in figures 1(b), (f), the $\omega^{(n)}$ arise from the discrete set of propagation constants $k_{x}^{(n)} \simeq \pi n / a N$ imposed by the boundary conditions for the finite structures with $n$ either even or odd.

The peaks in $\gamma_{\text {tot }}$ at the set of frequencies $\omega^{(n)}$ build up on top of a fairly constant background within the frequency range displayed in figures 4(b) and 5(b). We assume that this background represents the contribution of $\gamma^{\prime}$ to $\gamma_{\text {tot }}$, and subtract the background to estimate $\gamma_{1 \mathrm{D}}$. The resulting form for $\gamma_{1 \mathrm{D}}(\omega)$ consists of a set of resonant peaks whose heights at discrete $\omega^{(n)}$ qualitatively map out $\gamma_{1 \mathrm{D}}^{(\infty)}$ calculated for the infinite structure, with the maximum peak height for $\gamma_{1 \mathrm{D}}$ occurring for the peak closest to the band edge. Moving further away from the band edge, we find that our numerical estimate of $\gamma_{\mathrm{tot}}(\omega)-\gamma^{\prime}(\omega)$ asymptotes to the calculated value of $\gamma_{1 \mathrm{D}}^{(\infty)}(\omega)$ reasonably well.

\section{D.1. Estimation of $\gamma_{1 \mathrm{D}}$ and $\gamma^{\prime}$}

From the previous discussions, we identify that the decay rate into other modes $\gamma^{\prime}$ can be read off from the broad background in $\gamma_{\text {tot }}$. Specifically, we estimate $\gamma^{\prime}=\gamma_{\text {tot }}\left(\omega^{\prime}\right)$ at a frequency $\omega^{\prime}$ just across the band edge and away from any resonant peak for a guided mode. The decay rate into the probe mode $\gamma_{1 \mathrm{D}}$ can then be estimated using $\gamma_{1 \mathrm{D}}=\gamma_{\text {tot }}-\gamma^{\prime}$.

For the single-beam structure and the atomic spin orientation shown in figure 4(b), we find a peak total decay rate $\gamma_{\text {tot }} / \gamma_{0} \approx 15$ and a background level $\gamma^{\prime} / \gamma_{0} \approx 1.2$ near the cesium D2-line frequency $v_{\mathrm{A}}=352 \mathrm{THz}$. We estimate the coupling to the resonant probe mode $\gamma_{1 \mathrm{D}}=\gamma_{\mathrm{tot}}-\gamma^{\prime} \approx 14 \gamma_{0}$. For the double-beam structure and spin orientation shown in figure 5(b), 
we find $\gamma_{\text {tot }} / \gamma_{0} \approx 22, \gamma^{\prime} / \gamma_{0} \approx 1$, and, therefore, $\gamma_{1 \mathrm{D}} / \gamma_{0} \approx 21$. Here, $\gamma_{0} / 2 \pi=5.2 \mathrm{MHz}$ is the free-space (vacuum) decay rate for the $D 2$ line.

\section{Appendix E. Effective area and mode volume for probe}

Both the single and double nanobeam structures lead to atom localization in a region of large amplitude for the probe field. One measure of the strength of the atom-field coupling is the effective mode volume $V_{\mathrm{m}}$ per unit cell, where

$$
V_{\mathrm{m}}=\int \epsilon(\mathbf{r})|\mathbf{E}(\mathbf{r})|^{2} \mathrm{~d}^{3} r / \epsilon\left(\mathbf{r}_{\min }\right)\left|\mathbf{E}\left(\mathbf{r}_{\min }\right)\right|^{2} .
$$

Here the integration is carried out over the volume of a unit cell. That is, the integration domain along propagation direction $x$ extends over the distance $a$ (i.e. the lattice constant), while in the transverse $y, z$ directions, the integration domain is from $-\infty$ to $+\infty$.

For the single nanobeam, the probe mode has a global maximum at $\mathbf{r}_{\min }=0$ and an effective mode volume $V_{\mathrm{m}} \sim 0.13 \mu \mathrm{m}^{3}$. For the double nanobeams, the probe mode has a saddle-like intensity distribution around $\mathbf{r}_{\min }=0$, resulting in $V_{\mathrm{m}} \sim 0.11 \mu \mathrm{m}^{3}$ for a unit cell.

\section{References}

[1] Kimble H J 2008 Nature 4531023

[2] For a review see Vahala K J 2003 Nature 424839

[3] Lev B, Srinivasan K, Barclay P, Painter O and Mabuchi H 2004 Nanotechnology 15 S556

[4] Dayan B, Parkins A S, Aoki T, Ostby E P, Vahala K J and Kimble H J 2008 Science 3191062

[5] Lepert G, Trupke M, Hartmann M J, Plenio M B and Hinds E A 2011 New J. Phys. 13113002

[6] Volz J, Gehr R, Dubois G, Estve J and Reichel J 2011 Nature 475210

[7] Cirac J I, Zoller P, Kimble H J and Mabuchi H 1997 Phys. Rev. Lett. 783221

[8] Duan L-M and Kimble H J 2004 Phys. Rev. Lett. 92127902

[9] Shen J T and Fan S 2005 Opt. Lett. 302001

[10] Kien F L, Gupta S D, Nayak K P and Hakuta K 2005 Phys. Rev. A 72063815

[11] Dzsotjan D, Sørensen A S and Fleischhauer M 2010 Phys. Rev. B 82075427

[12] Kien F L and Hakuta K 2008 Phys. Rev. A 77013801

[13] Chang D E, Sørensen A S, Demler E A and Lukin M D 2007 Nature Phys. 3807

[14] Zoubi H and Ritsch H 2010 New J. Phys. 12103014

[15] Chang Y, Gong X R and Sun C P 2011 Phys. Rev. A 83013825

[16] Chang D E, Jiang L, Gorshkov A V and Kimble H J 2012 New J. Phys. 14063003

[17] Asboth J K, Ritsch H and Domokos P 2008 Phys. Rev. A 77063424

[18] Chang D E, Cirac J I and Kimble H J 2013 Phys. Rev. Lett. 110113606

[19] Ye J, Kimble H J and Katori H 2008 Science 3201734

[20] Ovchinnikov Y B, Shulga S V and Balykin V I 1991 J. Phys. B: At. Mol. Opt. Phys. 243173

[21] Vernooy D W and Kimble H J 1997 Phys. Rev. A 551239

[22] Burke J, Chu S T, Bryant G W, Williams C J and Julienne P S 2002 Phys. Rev. A 65043411

[23] Renn M J, Montgomery D, Vdovin O, Anderson D Z, Wieman C E and Cornell E A 1995 Phys. Rev. Lett. 753253

[24] Ito H, Nakata T, Sakaki K, Ohtsu M, Lee K I and Jhe W 1996 Phys. Rev. Lett. 764500

[25] Christensen C A, Will S, Saba M, Jo G B, Shin Y I, Ketterle W and Pritchard D 2008 Phys. Rev. A 78033429

[26] Bajcsy M, Hofferberth S, Balic V, Peyronel T, Hafezi M, Zibrov A S, Vuletic V and Lukin M D 2009 Phys. Rev. Lett. 102203902 
[27] Vetsch E, Reitz D, Sagué G, Schmidt R, Dawkins S T and Rauschenbeutel A 2010 Phys. Rev. Lett. 104203603

[28] Dawkins S T, Mitsch R, Reitz D, Vetsch E and Rauschenbeutel A 2011 Phys. Rev. Lett. 107243601

[29] Goban A, Choi K S, Alton D J, Ding D, Lacrožte C, Pototschnig M, Thiele T, Stern N P and Kimble H J 2012 Phys. Rev. Lett. 109033603

[30] Rosenbusch P, Ghezali S, Dzuba V A, Flambaum V V, Beloy K and Derevianko A 2009 Phys. Rev. A 79013404

[31] Kien F L, Schneeweiss P and Rauschenbeutel A 2013 Eur. Phys. J. D 6792

[32] Ding D, Goban A, Choi K S and Kimble H J 2012 arXiv:1212.4941

[33] Hinds E A, Lai K S and Schnell M 1997 Phil. Trans. R. Soc. A 3552353

[34] Agarwal G S 1975 Phys. Rev. A 121475

[35] Buhmann S Y, Knöll L, Welsch D G and Dung H T 2004 Phys. Rev. A 70052117

[36] Rodriguez A W, McCauley A P, Joannopoulos J D and Johnson S G 2009 Phys. Rev. A 80012115

[37] Rahi S J, Emig T and Kardar M 2010 Phys. Rev. Lett. 105070404

[38] Soljacic M, Johnson S G, Fan S, Ibanescu M, Ippen E and Joannopoulos J D 2002 J. Opt. Soc. Am. B 192052

[39] Koenderink A F, Kafesaki M, Soukoulis C M and Sandoghdar V 2006 J. Opt. Soc. Am. B 231196

[40] Manga Rao V S C and Hughes S 2007 Phys. Rev. Lett. 99193901

[41] For a review see Baba T 2008 Nature Photon. 2465

[42] Hoang T B, Beetz J, Midolo L, Skacel M, Lermer M, Kamp M, Hšfling S, Balet L, Chauvin N and Fiore A 2012 Appl. Phys. Lett. 100061122

[43] Joannopolous J D, Johnson S G, Winn J N and Meade R D 2008 Photonic Crystals: Molding the Flow of Light (Princeton, NJ: Princeton University Press)

[44] Johnson S G and Joannopolous J D 2001 Opt. Express 8173

[45] Eichenfeld M, Camacho R, Chan J, Vahala K J and Painter O 2009 Nature 459 550-6

[46] Søndergaard T and Tromborg B 2001 Phys. Rev. A 64033812

[47] Thompson J D, Tiecke T G, de Leon N P, Feist J, Akimov A V, Gullans M, Zibrov A S, Vuletić V and Lukin M D 2013 Science 340 1202-5

[48] Oskooi A F, Roundy D, Ibanescu M, Bermel P, Joannopoulos J D and Johnson S G 2010 Comput. Phys. Commun. 181687

[49] Buhmann S Y and Welsch D G 2007 Prog. Quantum Electron. 3151

[50] Cataldo G, Beall J A, Cho H M, McAndrew B, Niemack M D and Wollack E J 2012 Opt. Lett. 204200

[51] Djurisic A B and Li E H 1998 Appl. Opt. 375291

[52] Dzayloshinkii I E, Lifshitz E M and Pitaevskii L P 1961 Sov. Phys._Usp. 4153

[53] Contreras-Reyes A M, Guérout R, Neto P A M, Dalvit D A R, Lambrecht A and Reynaud S 2010 Phys. Rev. A 82052517

[54] Kien F L, Gupta S D, Balykin V I and Hakuta K 2005 Phys. Rev. A 72032509

[55] Hwang J K, Ryu H Y and Lee Y H 1999 Phys. Rev. B 60 4688-95

[56] Yao P, Manga Rao V S C and Hughes S 2010 Laser Photon. Rev. 4 499-516 